\title{
The Methodology and Application of New Three-dimensional Guide System and Intelligent Service in Intelligent Scenic Spot: A Case Study of Qixing Scenic Spot in Guilin City, Guangxi, China \\ Baishou Li ${ }^{1,2}$, Sikai $\mathrm{Wu}^{1}$,Xue $\mathrm{Li}^{1}$,Jiahao Guo ${ }^{1}$,Binglin $\mathrm{Zhu}^{1}$, Weiguo Yang ${ }^{1}$ \\ ${ }^{1}$ College of Geomatics and Geoinformation, Guilin University of Technology, Guilin, Guangxi, China - (lbszhb, wusikaizju, 1x1378156549, 18577364554,m18577327672_1,ywg0607)@163.com \\ ${ }^{2}$ Guangxi Key Laboratory of Spatial Information and Geomatics, Guilin, Guangxi, China - lbszhb@163.com
}

KEY WORDS: Intelligent Service, Three-dimensional Tour Guide System, Three-dimensional Map, 3D Symbol System

\begin{abstract}
:
Nowadays, 3D navigation systems and intelligent services are costly to use, and the accuracy of intelligent navigation does not meet the needs of tourists, and the practicality is not strong. Based on the current situation of the construction of the smart scenic navigation system and the actual needs of tourists, this study determines the main principles and methods of the tour guide system and intelligent services. Taking a certain scenic spot as the specific research object, the $\mathrm{A}^{*}$ algorithm is used to solve the path. By optimizing the online gracing service of the arc gis server network, the appropriate data interpolation method is used to improve the accuracy of the elevation data of the scenic spot, and the characteristics of the three-dimensional symbol system are combined. The 3D symbol design system is optimized, and a high-precision 3D navigation map model is established. Finally, the corresponding 3D tour guide system and scenic area intelligent service are designed based on this, and the principle and application flow of the intelligent scenic area navigation system construction are summarized. The three-dimensional map model and its navigation system designed by this research institute reduce the risk of tourists during the tour, improve the quality and efficiency of the tour, and provide a practical service optimization solution for the smart scenic three-dimensional navigation system. The construction and use cost of the high-precision three-dimensional navigation system in the scenic spot is reduced.
\end{abstract}

\section{INTRODUCE}

With the rapid development of the economy, the advancement of science and technology, the improvement of people's quality of life, and the continuous improvement of the national vacation system, tourism has become the main choice for people on holidays. Based on the background, the famous tourist attractions will have a huge flow of people during the holidays. Some of the attractions are likely to be crowd,which affects the tourist experience. The tourism industry is booming and the number of tourists is increasing. This brings more opportunities to the tourist attractions, but it also brings greater challenges .In essence, the main reasons for this situation are the problems which are within the scenic spot.Such as the management system is not rigorous, the defects of the service system, the scenic traffic facilities and the map navigation are not perfect enough to lead to the efficiency of the service visitors. Because the map navigation symbols are general, the map lacks terrain elements, and the three-dimensional map navigation system of the scenic spot has low precision, low practicability, and unclear identification of safety hazard areas, which is not conducive to tourists choosing efficient and reasonable navigation routes when visiting scenic spots. It can't help the scenic area to properly digest too crowded people in the case of huge human traffic, and to alleviate problems such as blockages. Establishing a systematic, networked and intelligent three-dimensional map navigation system for scenic spots has become an increasingly urgent need

\section{THREE-DIMENSIONAL NAVIGATION SERVICE SYSTEM}

\subsection{The Acquisition of scenic terrain data}

Building a navigation service system in the relevant area and obtaining local topographic data is the most basic but necessary task. The current methods of obtaining basic geographic information fall into three categories:

(1) Obtained from the map of the finished product. The scanner is used for scanning, and the scanned photos are registered according to the accurate coordinate points, and then the corresponding digital map is obtained by vectorization.

(2) Perform basic measurements using basic measuring 
instruments such as total stations and theodolites. The instrument directly measures the coordinates and elevation of the scenic spot, and finally the data processing and mapping on the computer.

(3) Use the existing network resources to download relevant geographic basic information data from the website. For example, elevation data can be downloaded from the Geospatial Data Cloud (http://www.gscloud.cn/) of the Computer Network Information Center of the Chinese Academy of Sciences.

Each of the three methods has its own characteristics. The first method can obtain two-dimensional coordinate data to construct a simple two-dimensional plane map, but it is difficult to obtain elevation data from it, and cannot construct a three-dimensional map model. The second way can obtain coordinates. Point data can also obtain elevation data, and the data accuracy is high, but the workflow is more complicated, including engineering bidding, acceptance, etc., time cost and capital consumption are too large, not worth the loss; the third way, the feasibility is relatively Higher, download elevation data from the relevant website, image data can be up to 30 meters.

\subsection{The Encryption of DEM data}

There are many encryption methods for current DEM data. The most commonly used and well-effected algorithms are genetic algorithms, ant colony algorithms, and the more classical $\mathrm{A}^{*}$ algorithms. The encryption methods applied to DEM data in different geographical areas are different. For example, some encryption methods tend to smooth the interpolation result, so it is not suitable for scenic spots with large regional elevation fluctuations. The error caused by different encryption methods is also different. When selecting the encryption method, the error size should be carefully compared, and the encryption method with smaller error should be selected to interpolate the DEM.

\subsection{Terrain modeling and satellite imagery}

After obtaining higher-precision elevation data and basic coordinate data, a three-dimensional terrain model can be constructed. The fineness of the model is determined by the scale of the elevation data and the grid size. Texture information can be obtained from related satellite imagery images, and related mapping operations can be performed in 3D software such as Arc Scene.

\subsection{Design and application of 3D symbol system}

After the construction of the textured 3D map, you need to add the necessary symbology to facilitate subsequent path selection and scenic tour. The designed three-dimensional symbol system should meet the actual needs, meet the tourist's navigation needs, and remind visitors to pay attention to the security situation in the adjacent area.

\subsection{Carry the appropriate path solving algorithm}

Similar to the case of selecting the DEM data encryption method, it is necessary to carefully compare the applicable range of each path solving algorithm and the interpretation of the path optimal solution when selecting the path navigation algorithm. Due to the different requirements of path solving, the path solving algorithms for the flat terrain urban areas and the mountainous terrain with large terrain fluctuations are often different. When selecting the solution algorithm, the weights of various demand situations should be allocated, and the path solving algorithm that is more suitable for the scenic spot should be selected.

\section{PATH SOLVING ALGORITHM}

In the scenic terrain of complex terrain, the road conditions and terrain fluctuations are more complicated. Obstacle and hidden points are doped in the scenic area, so we find an algorithm that is computationally efficient and can take advantage of 3D path planning. Among the existing problems of 3D path planning, the main research algorithms are $A^{*}$ algorithm, genetic algorithm, ant colony algorithm, based on minimum energy function method, particle swarm optimization algorithm and artificial potential energy algorithm. The genetic algorithm is to study Darwin's biological evolution theory to simulate the natural evolution process and the biological evolution process related to genetics. By establishing a computational model to search for the optimal solution, the genetic algorithm can directly operate on structural objects. Without the limitation of derivative and function continuity, the process is simple, but the search speed of genetic algorithm is slow, and it takes more time to get accurate solution; ant colony algorithm is a self-organizing, essentially parallel algorithm. It is a positive feedback process and has strong robustness. The ant colony algorithm is a probabilistic algorithm used to find the optimal path in the graph. The group optimization law through the foraging behavior of ant colony The bionic optimization mathematical model is established, but the ant colony algorithm 
is easy to lose the optimal solution, which is caused by the process of positive feedback in the algorithm. The minimum energy function method is mainly applied to the 3D path planning of unmanned autonomous vehicles or ground mobile robots. The question is whether the criterion of a certain path satisfies the minimum elastic energy of the curve as the criterion for the optimal path; the A* algorithm is one Heuristic search algorithm, and in the field of path optimization, $\mathrm{A}^{*}$ algorithm is playing an important role. For example, when people use electronic map navigation in scenic spots, they choose the starting point and the end point to get the navigation route, and the $\mathrm{A}^{*}$ algorithm is research. The shortest path problem from point to point including the specific path. The following are three algorithms that are more suitable for $3 \mathrm{D}$ path planning.

\subsection{Genetic algorithm}

Genetic algorithm (GA) is a global optimization probability search algorithm that simulates the inheritance and survival of the inferior rule in Darwin's biological evolution theory. It is an algorithm that mimics the natural genetic mechanism and the rule of survival of the fittest. In the early and late stages of the genetic evolution process, the probability of individual selection in the population will vary to varying degrees. The idea of genetic algorithm is to first select the candidate solution, then test the fitness of the candidate solution according to the established adaptive index, leaving the solution with higher fitness, and then repeat the above operation to make new The candidate solution is looped until the optimal solution is found.Genetic algorithms have strong robustness and good ability to search global optimal solutions, especially when studying the optimal combination of optimal paths. Another feature of the genetic algorithm is that it has better flexibility and adaptability, so it can find the global optimal solution, and it is difficult to fall into the local optimal solution. However, in the process of searching, the genetic algorithm needs to use a large amount of historical information to genetic algorithm, so this causes the algorithm to be similar to premature, low computational efficiency, etc., and also determines that the genetic algorithm has strong limitation. Therefore, in the case of a complex landscape with complex environment and rugged terrain, the shortcomings of the genetic algorithm's computational efficiency and stability are not high, and the ability to search for new paths is poor. It may be necessary to obtain multiple calculations at times. Stable results, which will undoubtedly consume a lot of time and economic costs, limit the wide application of genetic algorithms in intelligent scenic spots.

\subsection{Ant Colony Algorithm}

Ant colony algorithm is a probabilistic algorithm, which is mainly used to find the optimal path in the graph. This algorithm is also called ant algorithm. The ant colony algorithm is produced by its creators by observing the natural ant's process of finding food and using artificial ants to mimic the natural ant foraging behavior, so this is a bio-bionic algorithm. The core of this algorithm is because ants release a very important thing in the path during the foraging process pheromone. The behavior of the whole ant colony foraging will continue to affect the concentration of pheromones on each path. The more ants passing through the path, the higher the pheromone concentration, and vice versa, the lower the pheromone concentration, and the pheromone concentration. The higher the path is selected by ants, the higher the probability is, the so-called positive feedback process, but how does this process ensure that the path with the highest concentration of pheromone is the shortest path? In fact, it can be understood that when a path makes the food closer to the ant nest than the other path, the time for the ant to return to the nest through this path will be shorter. When the number of ants going back and forth is more and more, this path The pheromone concentration is significantly higher than the longer round trip time, and the path with the highest pheromone concentration is the optimal path from the nest to the food. In addition to the shortest path, this process provides several suboptimal paths. Therefore, each time the ant colony algorithm completes a related search, in addition to the optimal solution, it can provide several suboptimal solutions, which will The defect that causes the ant colony algorithm to fall into the local optimal solution will affect the choice of the shortest path to some extent.

\subsection{A* algorithm}

The now popular heuristic search algorithm should belong to the $\mathrm{A}^{*}$ algorithm, and the $\mathrm{A}^{*}$ algorithm is a direct search method. The so-called direct search method refers to the method of directly searching without any preprocessing. The difference between the algorithm is that the global information is introduced when searching for each possible node in the shortest path, and the distance between the current node and the end point is estimated, and used as a criterion to judge whether the node is in the On the shortest path. In addition, the $\mathrm{A}^{*}$ algorithm also introduces the evaluation function $\mathrm{f}(\mathrm{n})=\mathrm{g}(\mathrm{n})+$ 
$\mathrm{h}(\mathrm{n})$, where $\mathrm{g}(\mathrm{n})$ is the shortest path value known from the starting raster to the grid $\mathrm{n}, \mathrm{h}(\mathrm{n})$ is the valuation heuristic value of the grid $\mathrm{n}$ to the target. If the actual cost from $\mathrm{n}$ to the target is always greater than or equal to $\mathrm{h}(\mathrm{n})$, then the shortest path will definitely be found. For the three-dimensional $A^{*}$ algorithm h(n), the Euclidean distance is usually taken as the heuristic value because it is allowed to move in any direction in three-dimensional space. Since the actual distance from the grid $\mathrm{n}$ to the destination grid is generally larger than this heuristic value, it is guaranteed that the search path distance is the shortest.The $A^{*}$ shortest path search algorithm can be implemented by $\mathrm{C}$ language using $\mathrm{VC}++6.0$ software. The result of running in $\mathrm{VC}++$ is shown in the following figure. This is a simulated flat map. The blank squares represent the roads that can pass, and

the white squares are obstacles that are impassable (each grid in the program represents one step, Understand that the map is a $20 * 20$ matrix, and solve the path by inputting the coordinates of the starting point and the coordinates of the end point).

In Figure 1, "ם refers to a road that can pass, and "•" represents an obstacle, meaning that it is impassable.

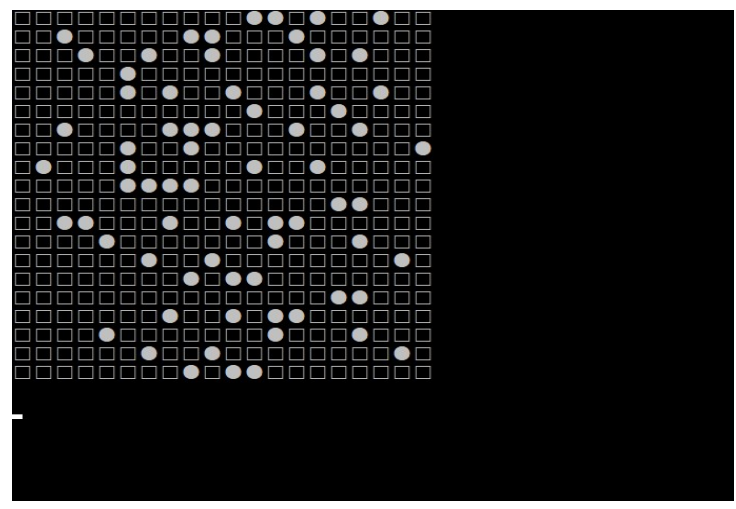

Figure 1

In Fig. 2, when the starting point coordinates $(1,1)$ and the ending point coordinates $(9,10)$ are input, the shortest path can be obtained, and the minimum number of steps can be obtained. "※" stands for the starting point, "ㅇ" stands for the end point, and " $\tau$ " stands for the shortest path.

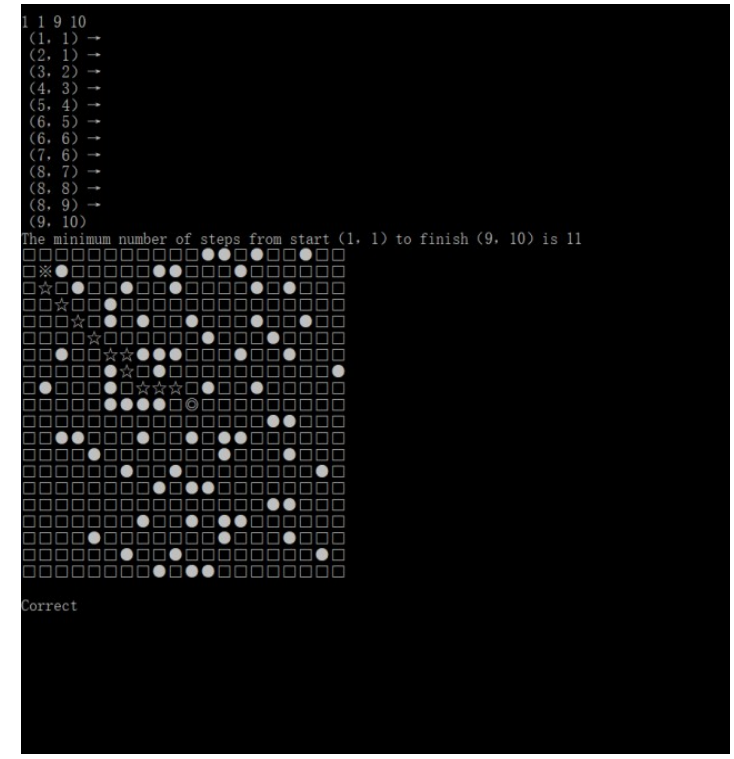

Figure 2

When the 3D path planning algorithm is applied to the path solving of the 3D scenic area model, many algorithms lose their advantages and even complete the solving task. The real scenic terrain is more complex, the road is rugged, and there are many hidden points and obstacles. When these inaccessible factors exist in the path, Then this path will not be feasible. Among the above several suitable algorithms, the $\mathrm{A}^{*}$ algorithm is more suitable for the path solving of the real scene, because the $A^{*}$ algorithm is more suitable for multiple obstacles, and the algorithm is fast and efficient, and the terrain is fluctuating. There are advantages in maps with many obstacles. Like the literature, the slope distance is equivalent to a horizontal distance, and a new $\mathrm{g}(\mathrm{n})$ representation method is introduced to improve the $\mathrm{A}^{*}$ algorithm, and the final practical application effect is ideal, which is very good for the three-dimensional scenic path planning. Great practicality.

\section{THE CHOICE OF DEM ENCRYPTION METHOD,} AND THE CONSTRUCTION OF 3D MAP MODEL

As an expression of digitization of terrain and geomorphol ogy, DEM accuracy is not easy to lose, and display meth ods are diverse. DEM has a wide range of applications, $s$ uch as geographic feature extraction, hydrological analysis, and three-dimensional display of surface information, the data is very functional. DEM is an indispensable basic dat a when building a 3D map model.Take the Qixing Scenic Spot in Guilin as an example. Currently, the DEM data $t$ hat we can download from the geospatial data cloud has a maximum precision of 30 meters. However, for the scen ic spots with large fluctuations, the model established by $t$ 
his precision is not practical and the reference value is no $\mathrm{t}$ high, and interpolation encryption is needed to meet the actual navigation requirements. The inverse distance weight (IDW) interpolation method, the global polynomial interpol ation method, the radial basis function (RBF) interpolation method, and the Kriging method are currently commonly used raster data interpolation methods. When the surface value changes drastically within a short distance or if the sample value is suspected of measurement error or uncerta inty, the global polynomial interpolation and Kriging interp olation will make the interpolated grid surface smooth, me asured and predicted. The error between them is relatively large, so it is not suitable for and applied to scenic spot $\mathrm{s}$ where the terrain changes more severely. The Kriging $\mathrm{m}$ ethod is similar to the interpolation result of the radial ba sis method, and the surface structure of the interpolated gr id structure is not much different. However, after compari ng the interpolation prediction charts of the two interpolati on methods, it is found that the radial basis interpolation method (RBF) has smaller errors and is more suitable for the scenic spots with more severe terrain changes.

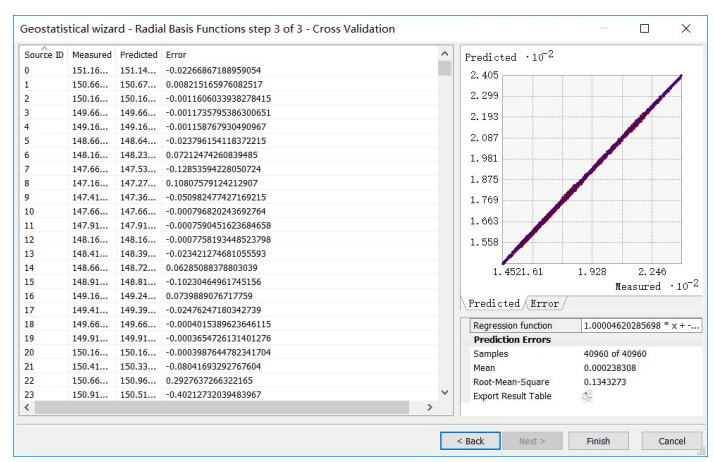

Figure 3

After encrypting the DEM data to match the raster resolution of the satellite imagery, the image can be applied to the texture map of the $3 \mathrm{D}$ terrain model. Satellite imagery contains geographic information in a certain area, and the information is rich, the effect is intuitive and realistic, and the acquisition method is simple. It can be used as the texture information of 3D map, which has certain reference value for the work of 3D modeling. After confirming that the satellite image and the DEM data are in the same projection zone, the model map can be implemented in the arc scene.

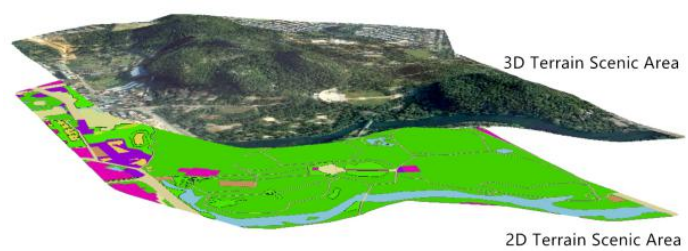

Figure 4

\section{DESIGN 3D SYMBOL SYSTEM}

Three-dimensional symbols are mainly divided into three types: point symbols, line symbols and surface symbols. Our 3D model symbols in ArcGIS software have the following unique features:

(1) Realization: It pursues model symbolization more than the design of ordinary $3 \mathrm{D}$ models, and the design is simple and visualized. Because of the special nature of the 3D simulation of ArcGIS software, the design of the 3D model is more about the external shape while expressing the most basic characteristics as much as possible. And material and color authenticity.

(2) Establishing contact: In the design process of the 3D model, it is usually necessary to establish an intuitive and intrinsic connection between the designed model and the model to be designed, so as to avoid designing each model symbol in isolation and one-sidedly. Fully considering the size, color, direction, transparency and texture of the object, the three-dimensional symbol designed can express the relevant geographic information well, optimize the effect of the three-dimensional landscape, and play a supporting role in the navigation process of the three-dimensional map model.

\section{ARC GIS SERVER MAP SERVICE OPTIMIZATI}

ON

\subsection{Hardware facilities}

Hardware facilities are critical to the operation of the software. Although the operating conditions of ArcGIS Server do not have clear hardware requirements, the quality of the software determines the speed of the software, and the processing speed of the server after the server is set up on the PC side. The high-quality hardware equipment can make a large number of map services. The gis network has higher operating efficiency. 
Improve hardware performance to optimize server information acceptance and processing.

\subsection{Network environment}

ArcGIS Server built by ArcObjects is a development tool $\mathrm{B} \backslash \mathrm{S}$ tool (browser/server). When the user uses the software, the browser sends an access request to the server on the other end of the Internet, and then the server receives the application signal and analyzes, calculates, and processes it. The information obtained after processing is fed back to the user. In this series of processes, a large amount of data is constantly being uploaded and downloaded, and the high speed and smoothness of the network environment is particularly important. Improve network speed, good network environment can improve server information reception and processing

\section{3 system layout}

The architecture of the ArcGIS Server system is a gis server, a web server, and a client. They can be on the same machine or on different machines. When the server receives less processing information, we can choose to have the gis server, web server and client deployed on the same machine. When the server is frequently accessed and the received data exceeds the load, the ArcGIS Server system components are laid out. On different machines, the purpose of optimizing the server information acceptance and processing process is achieved.

\subsection{Data Optimization}

6.4.1 Optimization of maps:Before the map service is rel eased, the map file data is processed, including the finishi ng of the layer, the optimization of the map document, th e use of annotation instead of the annotation, avoiding the appearance of very large text sizes, the use of simplified features, etc., which is beneficial to the release of the $\mathrm{m}$ ap. Users have faster access to download speeds.

6.4.2 Optimization of Map Cache: Map caching technolo gy, also known as tile technology, is based on a certain mathematical formula calculation principle. The map is div ided into certain standard pictures and stored in the comp uter disk. When the user starts the client browsing map se rvice, the server receives the information and goes straight to the current map. The "tiles" corresponding to the coor dinate positions, in order to reduce the load on the server, so that users can navigate the map more smoothly. The $\mathrm{t}$ hree formats JEPG, PNG32 and PNG8 are the mainstream of the map cache technology to save file formats. We ca $\mathrm{n}$ choose the format according to the characteristics of the file. The image file of the image slice is more inclined $t$ o use the JEPG format, the PNG8 is applicable to the ma $p$ file of the vector data slice, and the PNG32 can be use $\mathrm{d}$ for ordinary, no special image or vector slice. Choosing the right cache tile format can save storage space, provid e good use, and improve server operation efficiency.

\section{3D MAP SERVICE}

ArcGIS Pro is a professional desktop software developed by ESRI. It includes the main working modules of two-dimensional integration, mapping and visualization, task workflow, image management, spatial analysis and collaborative sharing. 3D capability is one of the important capabilities provided by ArcGIS Pro. It uses ArcGIS Pro to realize the browsing, editing and drawing of $2 \mathrm{D}$ and $3 \mathrm{D}$ data under the same project, and realize the complete linkage of $2 \mathrm{D}$ and 3D data. With ArcGIS Pro, you can browse, edit, analyze, and publish 3D data.

ArcGIS Online is a platform for visualization, analysis, collaboration, and application creation based on public cloud, allowing organizations to use, create, and share data, maps, and applications in Esri's secure cloud, as well as access to authoritative basemaps and ArcGIS applications. Programs that create, manage, and store data as published Web layers; allow administrators of organizations to manage members and groups of organizations, configure roles and application permissions for members, manage organizational quotas, and manage organizations Various information such as accounts. In ArcGIS Online, individuals, organizational members, and organizations can connect and collaborate with each other, and because it is a public cloud-based Web GIS platform, everyone can access ArcGIS Online anytime, anywhere, using any device.

The combination of the desktop software ArcGIS Pro and the online platform ArcGIS Online enables the entire process from the initial 3D model design to the ultimate browsing from the web and mobile terminals. DEM data interpolation and encryption, 3D model construction, model mapping, model release, and data online browsing can all be implemented in this.

\section{CONCLUSION}

With the rapid development of the tourism industry, the number of tourists continues to rise, tourists are increasingly demanding the intelligent service quality and navigation system 
of the scenic spot, and the two-dimensional map can no longer meet the individual needs of tourists. This paper integrates the actual needs of passengers and the current status of the construction of the navigation service system, and turns the research direction to the map guide design of the three-dimensional model.

The study takes a certain scenic spot as the specific research object. In the aspect of path optimization, based on the $A^{*}$ algorithm, combined with the straight line intersection algorithm in computer graphics, it is applied to the path search to improve the accuracy of the raster data path solution. In terms of service port, the online map service based on ArcGIS Server is adopted, and the information receiving and information processing process of the server is optimized. In the design of the guide system map model, the terrain data interpolation method is used to improve the data precision and establish a high-precision three-dimensional navigation map. Model; in the aspect of symbol system design, the three-dimensional symbol database is combined, and the basic characteristics of the three-dimensional symbol are summarized, and a concrete construction scheme is proposed for the three-dimensional guide system of the scenic spot.

The research and design of the guide system and the principle and application of intelligent service have reduced the risk of tourists during the tour. To a certain extent, it also improved the quality and efficiency of the tour, providing a high-precision map model design for the three-dimensional navigation system. The smart scenic three-dimensional tour guide system and its intelligent service provide a practical service optimization solution, which reduces the construction and use cost of the high-precision three-dimensional navigation system in the scenic spot to a certain extent.

\section{ACKNOWLEDGMENTS}

This work was supported by the Guilin Scientific Research and Technology Development Plan(20190601;2016012601), the Innovation and Entrepreneurship Training Program of Chinese College Students (NO.201910596257), Guilin University of Technology Ph.D Scientific research initial funding(002401003316);Chongqing basic science and advanced technology research (cstc2015jcyjBX0023).Special thanks to the College of Geomatics and Geoinformation, Guilin University of Technology for the support of our work.

\section{REFERENCES}

Zhong Jingwei., 2012. Design and Implementation of Scenic Spot System Based on RFID Technology. Modern Electronic Technology, 35(16): 8-11.

Xu Hui.,2013. Design and implementation of self-guided tour guide system based on Android in Urumqi. Fujian: Xiamen University

Liu Hanmei.,2012. Design and implementation of interactive intelligent tour guide system based on Android platform. Guangzhou: Jinan University,

LI Fei.,LIU Songlin., ZHAO Fulai., 2007. Research on Monitoring and Early Warning System of Scenic Spots Based on Web GIS.Beijing Surveying and Mapping,(1):26-28

Lu Xiping.,2018.Application of DEM Data Acquisition and Slope Analysis Based on Google Earth[J].Sichuan Water Resources, (5):101.

Liu Hengping.,2014. Searching for key molecular fingerprint fragments of anti-HBV active molecules based on genetic algorithm. Journal of China Pharmaceutical University, (4): 406.

Fan Tao.,2004.Application of computer technology and its optimization method in linear optimization design of route profile. Computer Application Branch of China Highway Society. Academic Papers of 2004 Annual Meeting of Computer Application Branch of China Highway Society. China Highway Society Computer Application Branch: China Highway Society,6.

Si Zhaolong., 2014.Design and implementation of remote intelligent home monitoring system based on ARM. Hangzhou University of Electronic Science and Technology

Li Xue., Li Baishou., Tang Shiqing.,Liao Mingzhi., Wu Sikai., Liang Shihai.,2019. GIS-based real-time optimization of the navigation route of intelligent scenic spots. China Science and Technology Information, (1): 208. 
LIU Zhengchun., JIANG Fukun.,2005.Research on $\beta$-model of optimal portfolio investment. Journal of Wenzhou Uni versity.

Chen Bingyan.,2012.Research on Optimum Path Selection Based on GIS. Dalian: Dalian Maritime University.

Li Xiongfei., Zhang Hailong., Liu Zhaojun., Wang Renxi., 2011.Solving the Shortest Path Problem with Heuristic Alg orithm. Journal of Jilin University (Engineering Edition) .

ZHAO De-qun., 2017.DUAN Jian-ying.,CHEN Peng-yu, SU Jin-hai., Optimal Path Planning of 3D Map Based on A* Algorithm. Journal of Computer Systems, (7): 146-152.

XIAO Tao.,2016.Study on Spatial Interpolation Method of Terrestrial Water Reserve Based on GRACE Data_-Taking Horqin Sandy Land as an Example.Surveying and Spatial Geography Information,39(12):22-23.

Fang Hui., 2007.Production process and quality control of digital orthophoto maps . Urban Survey, (3):70.

LI Guizhen.,YANG Defang.,YANG Xiaoying.,2015.Discussi on on Symbolization of 3D Geospatial Model.Surveying $\mathrm{T}$ echnique and Equipment,17(4):34-36

Song Li., Liang Dongye., Wei Xiandong.,2010.Data Optimi zation Strategy in Arcgis Application Development.Journal of Yangtze River Scientific Research Institute, 27(1): 42-4 4.

Long Qian., Zhou Xinzhi., 2007.Research on Web Application Based on ArcGIS Server Development.Journal of Chengdu University of Information Technology,22(6): 682-685.

Min Minjie., Zhang Qi., Shen Pingyue.,Zhao Qinglin.,2009. ArcGIS Server map caching technology and the applicatio $\mathrm{n}$ of Rest technology in meteorological services.Zhejiang Meteorology, 30:70-73.

ESRI China Information Technology Co., Ltd. 2019.ArcGI S 10.6 product white paper [Z]. ESRI China official webs ite http://www.esrichina.com.cn/index.html. 